\title{
AGING
}

\section{REST linked to longevity}

Zullo, J.M. et al. Nature 574, 359-364 (2019)

Many outstanding questions still need to be addressed to understand why we age. Most studies into the mechanisms of aging have focused on DNA damage, telomere shortening, loss of proteostasis, and mitochondrial dysfunction, but new theories are under investigation. A new study published in Nature combined the use of human data and of two model organisms to identify a conserved mechanism of aging that is mediated by neural activity and regulated by the transcription factor REST.

The investigators first analysed RNA sequencing and microarray data from the brain of aged individuals. They showed that genes related to neural excitation and synaptic function were downregulated in individuals $\geq 85$ years of age compared with individuals $\leq 80$ years of age, suggesting that extended longevity is associated with reduced neural circuit activity. Switching to the worm, the team showed that $\mathrm{Ca}^{2+}$ influx increased in ASH neurons during aging and that drug-mediated suppression of neural excitation extended C. elegans lifespan, suggesting that lifespan is dynamically regulated by the excitatory-inhibitory balance of neural circuits.

The investigators also suggest that the repressor function of REST is upregulated in the brain of individuals with extended longevity, resulting in downregulation of genes that mediate neural excitation. In the mouse, conditional KO of Rest increased neural activity in the brain compared with control, whereas in the worm CRISPR/cas9mediated induction of $s p r-4$, which encodes an orthologue of REST, reduced excitation in ASH neurons and extended lifespan.

The team also demonstrated that REST and neural activity converge with insulinIGF signalling (IIS) to regulate lifespan. RNA interference (RNAi)-mediated knockdown of daf-16, which encodes transcription factor DAF-16-the C. elegans homolog of FOXO acting downstream of IISprevented the extension of lifespan induced by $s p r-4$ overexpression. The extension of lifespan induced by RNAi of daf-2, which encodes IGF-1 receptor was also inhibited by mutations in $s p r-3$ and/or spr-4. These results suggest that $s p r-3$ and $s p r-4$ contribute to the regulation of lifespan via the IIS pathway in worms. The investigators also showed that SPR-3 and SPR-4 contribute to the extreme longevity of daf-2 mutants by repressing neural excitation, which leads to the activation of DAF-16.

"The activation of $d a f-16$ by REST orthologues in worms and FOXO1 by REST in humans might be a mechanism for integration of neural activity with metabolism" explain the investigators in the discussion, before concluding that activation of REST and reduction of excitatory neural activity could be an approach to slowing aging in humans.

\section{Alexandra Le Bras}

Published online: 9 December 2019 https://doi.org/10.1038/s41684-019-0448-x

\section{Take the PAiril Out of Pain Management}

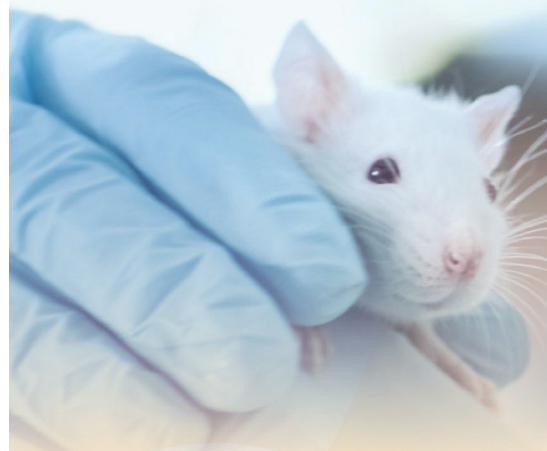

Pharmaceutical grade. FDA-indexed. Simple to order and administer.

NEW Ethiqa $X R^{\text {Tw }}$ is the first and only pharmaceutical grade extended-release buprenorphine, providing 72 hours of analgesia for mice and rats. Coming soon through distributors, Ethiqa XR will be simple to order, and its low-viscosity formula is easy to administer.

WARNING: Due to serious human safety and abuse concerns, read the entire product insert before using this drug, including the complete Box Warning.

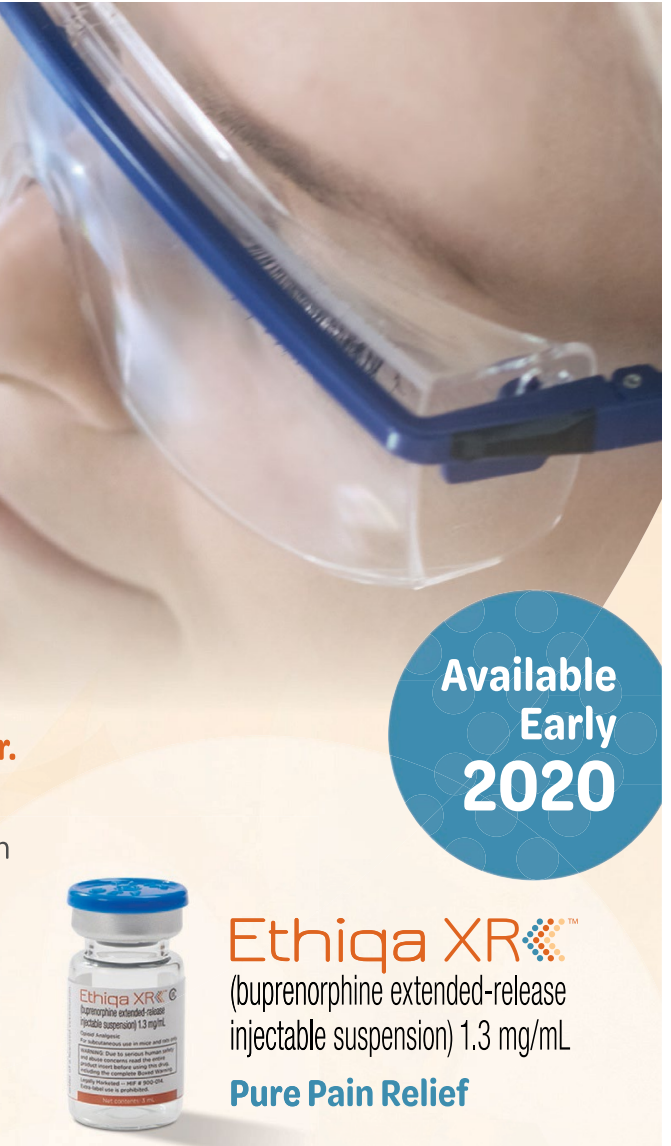

